\title{
Modelling and Optimizing Sequential Imperfect Preventive Maintenance
}

Michael Bartholomew-Biggs ${ }^{1}$, Ming J. Zuo ${ }^{2}$ and Xiaohu Li ${ }^{3}$

${ }^{1}$ School of Physics Astronomy and Mathematics, University of Hertfordshire, Hatfield, AL10 9AB, UK

2 Department of Mechanical Engineering, University of Alberta, Edmonton, Alberta, T6G 2G8, Canada

${ }^{3}$ School of Mathematics \& Statistics, Lanzhou University, Lanzhou, China 


\begin{abstract}
This paper deals with the problem of scheduling imperfect preventive maintenance (PM) of some equipment. It uses a model due to Kijima in which each application of PM reduces the equipment's effective age (but without making it as good as new). The approach presented here involves minimizing a performance function which allows for the costs of minimal repair and eventual system replacement as well as for the costs of PM during the equipment's operating lifetime. The paper describes a numerical investigation into the sensitivity of optimum schedules to different aspects of an age-reduction model (including the situation when parts of a system are non-maintainable - i.e., unaffected by PM.)
\end{abstract}

Key words Cost rate, Effective age, Failure mode, Failure rate, Imperfect preventive maintenance, Optimization 


\section{Introduction}

Most organizations incur significant costs associated with equipment failure and its subsequent repair or replacement. The frequency of such failure can typically be reduced by periodic maintenance. Mathematical models for analyzing and optimizing the performance of repairable equipment have been widely discussed in the literature [1] - [14]. In this paper we follow ideas given in [1] and [8] and study the optimal scheduling of preventive maintenance (PM), basing our approach on the notion that eqipment which benefits from PM can have an effective age which is less than its calendar age.

When only minimal repairs are performed and there are no other interventions, the likelihood of equipment failure can be expected to increase steadily with time. More precisely, we suppose that the number of failures occurring during a time interval $(a, b)$ is

$$
\int_{a}^{b} h(t) d t .
$$

The function $h(t)$ is sometimes called the failure rate or hazard rate (as in [8] or [10]) and sometimes the failure intensity $[4,5]$. If $H(t)$ denotes the indefinite integral $\int_{0}^{t} h(s) d s$, the number of failures occurring between $t=a$ and $t=b$ is $H(b)-H(a) . H(t)$ is called the cumulative failure rate.

In practice, preventive maintenance $(\mathrm{PM})$ is used to lengthen the useful lifetime of equipment (and hence to decrease average running cost) by reducing the occurrence of failures. One of the key characteristics of a maintenance model is the effect of different kinds of intervention on the age of the system. Perfect repair and minimal repair are both commonly used in idealised age-effect models; and similar terms can also be applied to maintenance. In reality, however, both repair and maintenance are usually imperfect i.e., somewhere between perfect and minimal. Pham and Wang [11] and, more recently, Doyen and Gaudoin [4] have given useful surveys of imperfect maintenance models. One of the most important of these is the effective age model (Kijima et al [6], [7]). This is also called the virtual age model. If we assume that maintenance makes the equipment's effective age, $y$, less than its calendar age, $t$, then the number of failures occurring after a PM will depend on $H(y)$ rather than $H(t)$. Since $H$ is a monotonically increasing function, fewer failures will occur after a PM than if PM had not been carried out.

Our purpose in this paper is to consider the optimal scheduling of preventive maintenance. Our particular focus is on the way that such schedules can be affected by the choice of aging model that is used. Specifically, we compare the so-called Type 1 and 
Type 2 aging models proposed in [6, 7]. These have also been recently discussed in an optimization context by Kahle [5].

\section{Effective age models}

In what follows we shall use $x_{k}$ to denote the interval between the $(k-1)$-th and $k$-th PM. Thus, if equipment enters service at time $t=0$, the first PM occurs at time $t_{1}=x_{1}$. Just before this maintenance, the effective age $y_{1}$ is the same as its calendar age $x_{1}$. Immediately after PM, however, the effective age is reduced to $b_{1} x_{1}$, where $b_{1}$ is some constant $\left(0<b_{1}<1\right)$. (We note here that we are making the idealised assumption that time taken to perform PM is negligible.) Then, until the next PM at time $t_{2}=x_{1}+x_{2}$, the effective age is $y=b_{1} x_{1}+x$ for $0<x<x_{2}=t_{2}-t_{1}$. In particular, we denote the effective age just after the first PM by $y_{1}^{+}=b_{1} x_{1}$. The effective age of the system just before the second PM at time $t_{2}$ is then $y_{2}=b_{1} x_{1}+x_{2}$.

After the second (and subsequent) PMs, the effective age reduction can be modelled in two different ways [2, 7]. In type 1 effective age reduction [7] it is assumed that, immediately after the second PM, the effective age becomes

$$
y_{2}^{+}=y_{1}^{+}+b_{2} x_{2}=b_{1} x_{1}+b_{2} x_{2}=y_{2}-\left(1-b_{2}\right) x_{2} \quad \text { where } 0<b_{1} \leq b_{2}<1 \text {. }
$$

More generally, between the $(k-1)$-th and the $k$-th PM, the effective age is

$$
y=b_{k-1} x_{k-1}+\cdots+b_{1} x_{1}+x
$$

where $0<x<x_{k}=t_{k}-t_{k-1}$ and $b_{1} \leq b_{2} \leq \ldots \leq b_{k} \leq 1$. Thus the effective age immediately after the $(k-1)$-th PM is

$$
y_{k-1}^{+}=y_{k-1}-\left(1-b_{k-1}\right) x_{k-1} .
$$

In type 2 effective age reduction [7] it is assumed that the effective age immediately after the second PM is $y_{2}^{+}=b_{2} y_{2}=b_{2} b_{1} x_{1}+b_{2} x_{2}$. More generally, between the $(k-1)$-th and the $k$-th PM, the effective age is

$$
y=b_{k-1} y_{k-1}+x=\left(b_{k-1} \cdots b_{2} b_{1}\right) x_{1}+\cdots+b_{k-1} x_{k-1}+x
$$

where $0<x<x_{k}=t_{k}-t_{k-1}$ and $b_{1} \leq b_{2} \leq \ldots \leq b_{k} \leq 1$. In particular, the effective age just after the $(k-1)$-th PM is

$$
y_{k-1}^{+}=b_{k-1} y_{k-1} \text {. }
$$


More compactly, (3) can be written $y=B_{k-1} x_{k-1}+B_{k-2} x_{k-2}+\ldots+B_{1} x_{1}+x$ where $0<x<x_{k}=t_{k}-t_{k-1}$ and $B_{j}$ denotes the product $b_{k-1} b_{k-2} \ldots b_{j}$.

Type 1 age-reduction has been investigated by Kijima and co-workers in [6, 7] while the type 2 model has also been discussed by Dagpunar [2] and Lin et al. [8]. Both types are considered in the recent papers by Doyen and Gaudoin [4] and Kahle [5]. The main difference between these effective age reduction models is as follows. In the type 1 model, the $k$-th PM makes an effective age reduction only as regards the actual aging of the system since the $(k-1)$-th PM. In the type 2 model, however, each PM is assumed to cause an effective decrease in all the aging that has taken place since time $t=0$. Hence, under the type 2 model, repeated PMs can have a cumulative age-reduction effect which does not occur with the type 1 model.

We could say that the type 2 model takes a more optimistic view of the benefits of PM. If we suppose that PM occurs annually then, for both the type 1 and type 2 aging models, the effective age after maintenance at the end of the first year is $b_{1}(<1)$ years. Hence both models predict the same number of failures in year two. However the effect of PM at the end of year two is dependent on which aging model is used. The type 1 effective age after the second PM is $b_{1}+b_{2}$ years; but the type 2 effective age is $\left(b_{1}+1\right) b_{2}$ years. If $b_{1}=b_{2}=0.5$, say, then the type 1 and type 2 effective ages after the second PM are, respectively, 1 year and 0.75 years. Thus, during year three, the type 2 model implies failures than the type 1 model. This difference will become even more marked in subsequent years.

Extra parameters can be included in both age-reduction models to make them reflect the complexities of a real system. In practice, after a number of PMs have been performed, equipment may be less robust than its effective age suggests; and we can model this using variable scaling factors on $h(t)$. We suppose the number of failures occurring in $\left(0, t_{k}\right)$ can be written as

$$
H\left(t_{k}\right)=\int_{0}^{y_{1}} h(y) d y+\sum_{j=1}^{k-1}\left\{\int_{y_{j}^{+}}^{y_{j+1}} A_{j} h(y) d y\right\}=\int_{0}^{y_{1}} h(y) d y+\sum_{j=2}^{k} H_{j-1}
$$

where

$$
H_{j-1}=\int_{y_{j-1}^{+}}^{y_{j}} A_{j-1} h(y) d y
$$

and where the $A_{j}$ are constants such that $1 \leq A_{1} \leq A_{2} \leq \ldots$ (The expression (5) can also be extended to make a distinction between maintainable and non-maintainable failure modes (see [8]). This will be considered in a later section.) 
The PM schedules presented in [8] are optimized by a semi-analytic solution technique which takes advantage of the relatively simple forms (Weibull functions) chosen for the function $h(t)$. Bartholomew-Biggs et al. [1] have considered the same type 2 age-reduction model as used in [8] but their PM schedules are optimized using general-purpose nonlinear minimization algorithms. Such techniques may be more suitable when the $h(t)$ are more complicated than Weibull functions. The problem formulations in [1] also feature constraints to exclude spurious solutions with unacceptably short (or even negative) intervals between PM. The main purpose of the present paper is to compare type 1 and type 2 age-reduction models when used to determine optimum PM schedules in a framework similar to that described in [1].

Before we proceed to formulate an optimization problem we shall list some general assumptions and notation.

\section{General assumptions}

The system enters service at time $t=0$.

When a system failure occurs, minimal repair takes place instantly.

Preventive maintenance is completed instantly.

The system may have two categories of failure modes, i.e., maintainable and non-maintainable.

The failure rate for non-maintainable parts of the system is not affected by minimal repair, preventive maintenance or system failure.

The failure rate for maintainable parts of the system is not changed by minimal repair but it is changed whenever a PM is performed.

\section{Notation}

$t_{k}=$ time duration from $t=0$ to the time of the $k$ th PM.

$x_{k}=t_{k}-t_{k-1}=$ interval between the $(k-1)$-th and $k$-th PM.

$y_{k}=$ effective age of the system just before $k$-th PM.

$y_{k}^{+}=$effective age of system just after $k$-th PM.

$N=$ total number of PM performed. (The $N$-th PM is a system replacement.)

$h_{a}(t)=$ failure rate of maintainable components

$h_{b}(t)=$ failure rate of non-maintainable components

$H(t)=$ cumulative failures up to time $t$ when no PM occurs. See (16).

$A_{k}=$ adjustment factor on $h_{a}(t)$ due to the $k$-th PM. $A_{k} \geq 1$.

$b_{k}=$ effective age reduction factor due to the $k$-th PM. $b_{k} \leq 1$.

$H_{j-1}(t)=$ cumulative failures from $t_{j-1}$ to $t$ when PM occurs $\left(t_{j-1} \leq t \leq t_{j}\right)$. See (6).

$c_{p}=$ cost of a PM. 
$c_{m}=$ cost of a minimal repair.

$c_{r}=$ cost of a system replacement.

$\gamma_{m}=$ relative maintenance $\operatorname{cost} c_{m} / c_{p}$

$\gamma_{r}=$ relative repair cost $c_{r} / c_{p}$

\section{Optimizing a PM schedule}

To optimize PM scheduling we minimize a performance function of the form

$$
C=\frac{R_{c}}{t_{N}}=\frac{\gamma_{r}+(N-1)+\gamma_{m} \sum_{j=1}^{N} H_{j-1}\left(t_{k}\right)}{t_{N}}
$$

where

$$
\gamma_{r}=\frac{c_{r}}{c_{p}} \text { and } \gamma_{m}=\frac{c_{m}}{c_{p}}
$$

The function $C$ is given in [8] and assumes that PM takes place $N-1$ times with the $N$-th PM actually being a replacement. The numerator $R_{c}$ in $(7)$ represents equipment lifetime cost, expressed as a multiple of the cost of one PM. $R_{c}$ includes the fixed costs of replacement and $N-1 \mathrm{PMs}$ plus the repair costs predicted by the failure-rate function. The denominator, $t_{N}$, is simply the total life of the equipment (since we assume that the $N$-th PM represents a replacement). Hence (7) represents the mean lifetime cost.

We can use (5) to write the numerator of $C$ in terms of effective age. Then, by means of either (1) and (2) or (3) and (4), we can express it in terms of the $x_{k}$ (intervals between the $k$-th and $(k-1)$-th PMs). Furthermore, $t_{N}=x_{1}+x_{2}+\ldots+x_{N}$ and so the minimization of the cost function (7) can be carried out using $x_{1}, \ldots, x_{N}$ as independent variables. Hence, for any chosen value of $N$, we can use a nonlinear optimization technique to find the optimal PM intervals to minimize the mean lifetime cost. In the examples which follow we adopt a transformation proposed in [1] and minimize a function $\tilde{C}(u)$ where $x_{i}=u_{i}^{2}$. This transformation prevents the iterative optimization methods from being attracted to spurious solutions with some $x_{i}<0$. The minimizations were performed using a Newton method and with first and second derivatives of $\tilde{C}$ being obtained by automatic differentiation as described in [1].

The choice of optimization method is not particularly relevant to our present purpose of exploring the sensitivity of optimum PM schedules to changes in the aging model. It is worth stating, however, that minimization of the mean cost function does not appear to be particularly difficult. The Newton method typically converges in quite a small number 
of iterations, even when a good initial estimate of the solution is not provided. We have found no evidence that the problems have multiple solutions.

\section{Qualitative effects of age-models on PM schedules}

Before carrying out our numerical investigation, we look more closely at the way that the two aging models can be expected to influence the optimization of a PM schedule. In particular, we argue that the type 2 model may lead to schedules with PM bunched together more closely than would be the case with the type 1 model.

To substantiate the previous remark we suppose that a double $P M$ is performed in which one maintenance is scheduled immediately after another. Under the type 1 model, the second PM would cause no decrease in effective age since the age-reduction factor would only be applied to the zero time elapsed since the previous PM. This is quite a reasonable model of reality: if routine adjustments and replacements have just been made we would not expect to improve the health of the system by doing the same operations all over again. The type 2 model, on the other hand, does imply that it can be beneficial to perform a double PM. If the $k$-th PM reduces effective age to $y_{k}^{+}=b_{k} y_{k}$ then a second PM performed immediately would decrease the effective age again to $y_{k+1}^{+}=b_{k+1} y_{k}^{+}=$ $b_{k+1} b_{k} y_{k}$. Indeed if we were to perform a $p$-tuple $P M$ - i.e. doing $p$ successive PMs with no time in between - then we could drive the effective age of the system arbitrarily close to zero! The practical benefits of such a strategy might of course be offset by cost considerations; but the fact that such unlimited rejuvenation appears possible does suggest that the type 2 model may give a less realistic picture of system behaviour than the type 1 model.

In the above discussion, however, we have ignored the potential role of the parameters $A_{j}$ in equation (5). Setting each $A_{j}>1$ can help to counteract any tendency in the type 2 model to encourage use of repeated PMs to drive effective age towards zero. Let us suppose that the cumulative failure rate, $H(t)$, is approximated by a quadratic function

$$
H(t)=c_{2} t^{2}+c_{1} t
$$

and that the effective age just after the $k$-th PM is $b_{k} y_{k}$. Then, by (5), the rate of increase of the function $H$ just after the PM is

$$
h_{k}=A_{k}\left(2 c_{2} b_{k} y_{k}+c_{1}\right)
$$


If the $(k+1)$-th PM is performed right after the $k$-th then, under the type 2 model, the effective age is further reduced to $b_{k+1} b_{k} y_{k}$ and the subsequent rate of increase of $H(t)$ is

$$
h_{k+1}=A_{k+1}\left(2 c_{2} b_{k+1} b_{k} y_{k}+c_{1}\right) \text {. }
$$

Hence the extra PM can yield an unrealistic benefit unless $h_{k+1} \geq h_{k}$, i.e.

$$
A_{k+1}\left(2 c_{2} b_{k+1} b_{k} y_{k}+c_{1}\right) \geq A_{k}\left(2 c_{2} b_{k} y_{k}+c_{1}\right)
$$

which will occur if

$$
A_{k+1} \geq A_{k} \frac{q_{k}+1}{b_{k+1} q_{k}+1} \quad \text { where } \quad q_{k}=\frac{2 c_{2} b_{k} y_{k}}{c_{1}} .
$$

We emphasise that the relationship (9) is only relevant to the type 2 aging model and it suggests that we should not use the simple choice $A_{k}=1$ for all $k$. There appears, however, to be no comparable objection to using constant $A_{k}=1$ with type 1 aging.

In a later section we shall consider some particular choices of $A_{k}$.

\section{Quantitative effects of age models on PM schedules}

In oerder to perform a numerical investigation of PM schedules under different aging models we require an expression for the cumulative failure rate function, $H(t)$. For illustrative purposes we have obtained two polynomial forms for $H(t)$ by considering the behaviour of an imaginary system for which - in the absence of maintenance - the cumulative probability of failure by the $k$-th month of operation is $k \times 0.25 \%$. By running simulations for such a system over a five-year period we obtained data for the average number of failures as a function of time. Least-squares polynomials were then constructed to approximate this data. (We imposed some restrictions on the polynomials - namely that the constant term is zero and the linear term has a positive coefficient - to ensure that $H(t)$ predicts zero failures at time $t=0$ and does not allow spurious negative failures when $t$ is near zero.) Two of the resulting expressions for $H(t)$ are as follows (where $t$ is in years):

Case A - quadratic

$$
H(t)=0.1676 t^{2}+0.0704 t
$$

Case B - cubic

$$
H(t)=-0.0036 t^{3}+0.1919 t^{2}+.0323 t
$$

This derivation of (10), (11) from simulated data means the $H(t)$ do have a certain degree of realism. We note that the least squares approach yields a polynomial form for $H(t)$ 
rather than the commonly-used Weibull form $\alpha t^{\beta}$. We acknowledge, of course, that for a real-life system it may be much more difficult to find functions $H(t)$ to model the failure rate. Practical techniques for gathering and using data to derive formulae for predicting system failures are of great importance; but they are outside the scope of this paper.

In most of our numerical experiments we shall treat the age-reduction factors $b_{j}$ in equations (2) and (4) as constants. Furthermore we shall assume that all the $A_{j}$ in (5) depend on a single parameter. Thus we have, for $j=1,2,3 \ldots$,

$$
b_{j}=\hat{b} \quad \text { where } \quad 0<\hat{b}<1 \quad \text { and } \quad A_{j}=\hat{a}^{j-1} \quad \text { where } \hat{a} \geq 1 \text {. }
$$

The purposes of our investigation include:

- to compare optimum PM schedules obtained with type 1 and type 2 aging;

- to see how schedules vary with the effectiveness of PM (as described by values of $\hat{b}$ and $\hat{a}$ in $(12))$;

- to consider how schedules are affected by the relative costs $\gamma_{m}$ and $\gamma_{r}$ in (8).

Most of our results will be obtained with the quadratic model of failure rate (10); but there will be a brief discussion of the alternative expression (11). While acknowledging that our experiments involve artificial problems we argue that the results will give useful indications of how optimal PM schedules are affected by the modelling of effective age.

\subsection{Comparing schedules based on type 1 and type 2 aging}

We now show how optimal PM solutions can differ depending on which aging model is used in forming the cost function (7). The results are based on the quadratic form (10) for $H(t)$, together with the following values for the parameters defined in (8) and (12):

$$
\gamma_{r}=500, \quad \gamma_{m}=100, \quad \hat{b}=0.5, \quad \text { and } \hat{a}=1.1
$$

In the notation used in (7), the number of PMs is $N-1$ and so minimizing $C$ with $N=1$ gives the time to replacement, $t_{N}^{*}$, which without any maintenance produces the least mean operating cost. For the cost data in $(13)$ we get $C^{*} \approx 190.1$ and the corresponding $t_{N}^{*}=x_{1} \approx 5.46$ years.

Table 1 is obtained by minimizing (7) with $N=2,3,4$ and shows how mean cost can be improved by preventive maintenance. For each $N$, we quote the optimal inter-PM times, $x_{k}$, the time to replacement, $t_{N}^{*}$ and the mean cost, $C^{*}$, using both the type 1 and the type 2 aging models. For a single application of PM, the two aging models are equivalent; but for two or more PMs they give different results. Broadly speaking, the type 2 model 
causes the first PM to be performed later than for a type 1 solution. Subsequent inter-PM intervals tend to be shorter for type 2 solutions than for type 1 . The type 1 model offers only one chance to undo the aging that occurs prior to the first PM; but in the type 2 model this can be offset by all subsequent PMs.

\begin{tabular}{|c|c|c|c|c|}
\hline no of PMs & & inter-PM times (years) & $t_{N}^{*}$ & $C^{*}$ \\
\hline \multirow[t]{2}{*}{1} & Type 1 & $3.39 \quad 2.73$ & 6.12 & 171.0 \\
\hline & Type 2 & $3.39 \quad 2.73$ & 6.12 & 171.0 \\
\hline \multirow[t]{2}{*}{2} & Type 1 & $\begin{array}{lll}2.68 & 2.15 & 1.48\end{array}$ & 6.32 & 166.6 \\
\hline & Type 2 & $\begin{array}{lll}3.13 & 1.27 & 2.28\end{array}$ & 6.67 & 158.2 \\
\hline \multirow[t]{2}{*}{3} & Type 1 & $\begin{array}{llll}2.43 & 1.95 & 1.34 & 0.66\end{array}$ & 6.37 & 165.9 \\
\hline & Type 2 & $\begin{array}{llll}2.94 & 1.19 & 1.08 & 1.93\end{array}$ & 7.13 & 149.1 \\
\hline \multirow[t]{2}{*}{4} & Type 1 & $\begin{array}{lllll}2.43 & 1.95 & 1.34 & 0.66 & 0.0\end{array}$ & 6.37 & 166.0 \\
\hline & Type 2 & $\begin{array}{lllll}2.8 & 1.13 & 1.02 & 0.92 & 1.65\end{array}$ & 7.53 & 142.2 \\
\hline \multirow[t]{2}{*}{5} & Type 1 & $\begin{array}{llllll}2.43 & 1.95 & 1.34 & 0.66 & 0.0 & 0.0\end{array}$ & 6.37 & 166.2 \\
\hline & Type 2 & $\begin{array}{llllll}2.69 & 1.09 & 0.98 & 0.89 & 0.8 & 1.43\end{array}$ & 7.8 & 137.0 \\
\hline
\end{tabular}

Table 1: Optimal costs based on Type 1 and Type 2 aging models

It is not particularly helpful to compare the values of $C^{*}$ for type 1 and type 2 solutions in Table 1 since the corresponding functions (7) are different. Hence we cannot say that the type 2 solutions are better than those for the type 1 model. It is more meaningful, however, to consider how the patterns of type 1 and type 2 optimal PM schedules differ. For type 1 schedules the inter-PM times, $x_{1}, x_{2}, \ldots, x_{N}$ form a decreasing sequence; but in type 2 solutions it is more common to have $x_{1}>x_{2}, \ldots>x_{N-1}$ followed by $x_{N-1}<x_{N}$. Under the type 2 aging model, the effective age after the last PM is typically much smaller than it is for a type 1 solution and hence it is economical to operate the system over a longer final period up to its replacement time, $t_{N}^{*}$.

For the case with $N=4$, Figures 1 and 2 plot effective age against elapsed time for the two age models. It is clear that the type 2 model leads to schedules in which the effective age has a downward trend so that $y_{k}^{+}<y_{k-1}^{+}$. This does not happen in the type 1 solutions in which effective age tends to increase even though it lags behind calendar age.

Table 1 shows that, for both models, the mean cost decreases (and $t_{N}^{*}$ increases) as the number of PMs increases from one to three. However, this trend does not continue 


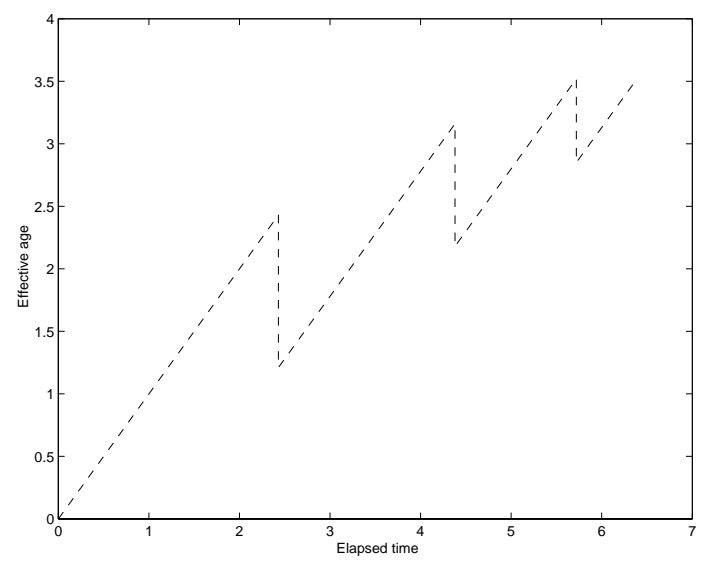

Figure 1: Effective age vs elapsed time for type 1 aging

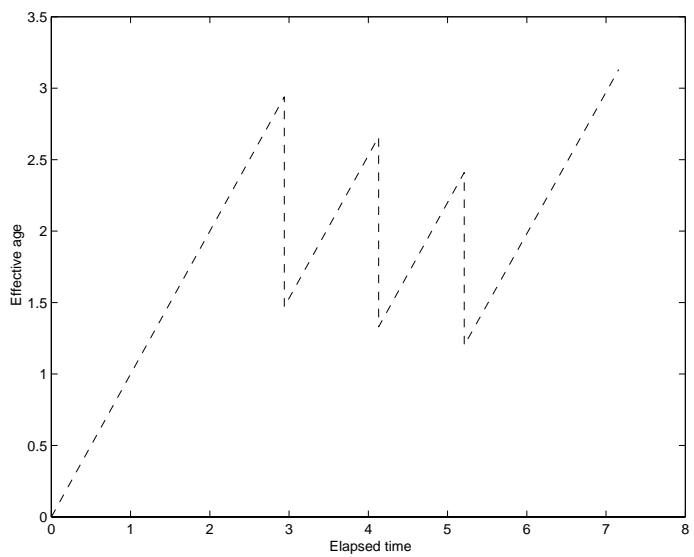

Figure 2: Effective age vs elapsed time for type 2 aging

for the type 1 model and it is not beneficial to do more than three PMs. If we optimize (7) for $N>4$ the optimal PM schedule remains essentially the same as in the last type 1 row in Table 1 because all the extra inter-PM times $x_{5}, x_{6}, \ldots$ are set to zero.

With the type 2 aging model it is advantageous to do more than three PMs. If we minimize ( 7 ) for $N=5,6, \ldots$, we find that $C^{*}$ continues to decrease until it reaches a minimum of about 116 when $N=23$ (which implies 22 applications of PM with $t_{N}^{*} \approx 10.3$ years). In this schedule, the initial PM is performed after about 2.3 years, while the second occurs less than a year later and subsequent inter-PM times continue to decrease steadily. Eventually the PMs take place every month or so (which might well be undesirable in practice). This type 2 schedule for $N=23$ may reflect the behaviour discussed in section 
4 - i.e., the possibility that a type 2 model can make the equipment appear arbitrarily young if double (or even $p$-tuple) PMs are performed. This rather unrealistic feature needs to be offset by the use of suitably large values for the $A_{j}$ parameters in equation (5); and we shall consider this point again in the next section.

\subsection{Effect of the parameter $\hat{a}$}

An increase in the value of $\hat{a}$ from 1 represents a decrease in the effectiveness of each PM, because, through (12), it increases the multiplying factors $A_{j}$ in equation (5). We have already pointed out that the parameters $A_{j}$ interact with type 1 and type 2 aging models in different ways. In particular, the inequality (9) is relevant to the type 2 model if we want to avoid schedules which perform many PMs close together to try and make virtually unlimited reductions in effective age.

If we consider the quadratic expression (10) for $H(t)$, then the parameter $q$ in $(9)$ is given by $q \approx 2.38 y_{k}$. If $b_{k+1}=b_{k}=0.5$ as in (13) then (9) becomes

$$
A_{k+1} \geq A_{k} \frac{2.38 y_{k}+1}{1.19 y_{k}+1}
$$

For the example problem considered above, the type 2 optimal schedules have an effective age $y_{1} \approx 2.5$ years just before the first PM. Subsequent $y_{k}$ decrease steadily to about 0.1 years when $k \approx 20$. Hence $(9)$ implies

$$
A_{k+1} \geq \rho A_{k} \quad \text { where } 1.75 \geq \rho \geq 1.1 \text {. }
$$

Such large values of the $A_{k}$ are probably not needed with type 1 aging, however, since this model automatically restricts the decrease in effective age that can be achieved by a single PM (or even by $p$-tuple PMs).

The examples which follow involve a benchmark case with 6 PMs (i.e. $N=7$ ). We keep the values of $\hat{b}, \gamma_{r}$ and $\gamma_{m}$ from (13) and only vary $\hat{a}$. In view of the remarks in the previous paragraph we use different ranges of $\hat{a}$ for the type 1 and type 2 age models. Specifically, we consider $1 \leq \hat{a} \leq 1.1$ for type 1 aging and $1.1 \leq \hat{a} \leq 1.75$ for type 2 .

The results in Table 2 were obtained with the type 1 aging model and they show $C^{*}$, the inter-PM times $x_{k}^{*}$ and $t_{N}^{*}$ for varying $\hat{a}$. The graphs in Figure 3 show how effective age varies with elapsed time for each of the $\hat{a}$ values.

The first row of Table 2 shows that the optimal schedule with $\hat{a}=1$ gives equally spaced PM intervals. As $\hat{a}$ increases we see, as would be expected, that $C^{*}$ also increases while $t_{N}^{*}$ decreases. 


\begin{tabular}{c|c|ccccccc|c}
$\hat{a}$ & $C^{*}$ & \multicolumn{1}{|c|}{$x_{k}^{*}$} & $t_{N}^{*}$ \\
\hline 1 & 146.3 & 1.04 & 1.04 & 1.04 & 1.04 & 1.04 & 1.04 & 1.04 & 7.27 \\
1.01 & 149.2 & 1.15 & 1.12 & 1.09 & 1.04 & 0.98 & 0.91 & 0.84 & 7.13 \\
1.025 & 153.3 & 1.35 & 1.27 & 1.17 & 1.04 & 0.88 & 0.71 & 0.53 & 6.94 \\
1.04 & 156.9 & 1.59 & 1.45 & 1.26 & 1.03 & 0.77 & 0.49 & 0.20 & 6.78 \\
1.05 & 158.9 & 1.77 & 1.58 & 1.32 & 1.02 & 0.68 & 0.32 & 0.0 & 6.69
\end{tabular}

Table 2: Optimal costs for type 1 schedules and varying $\hat{a}$
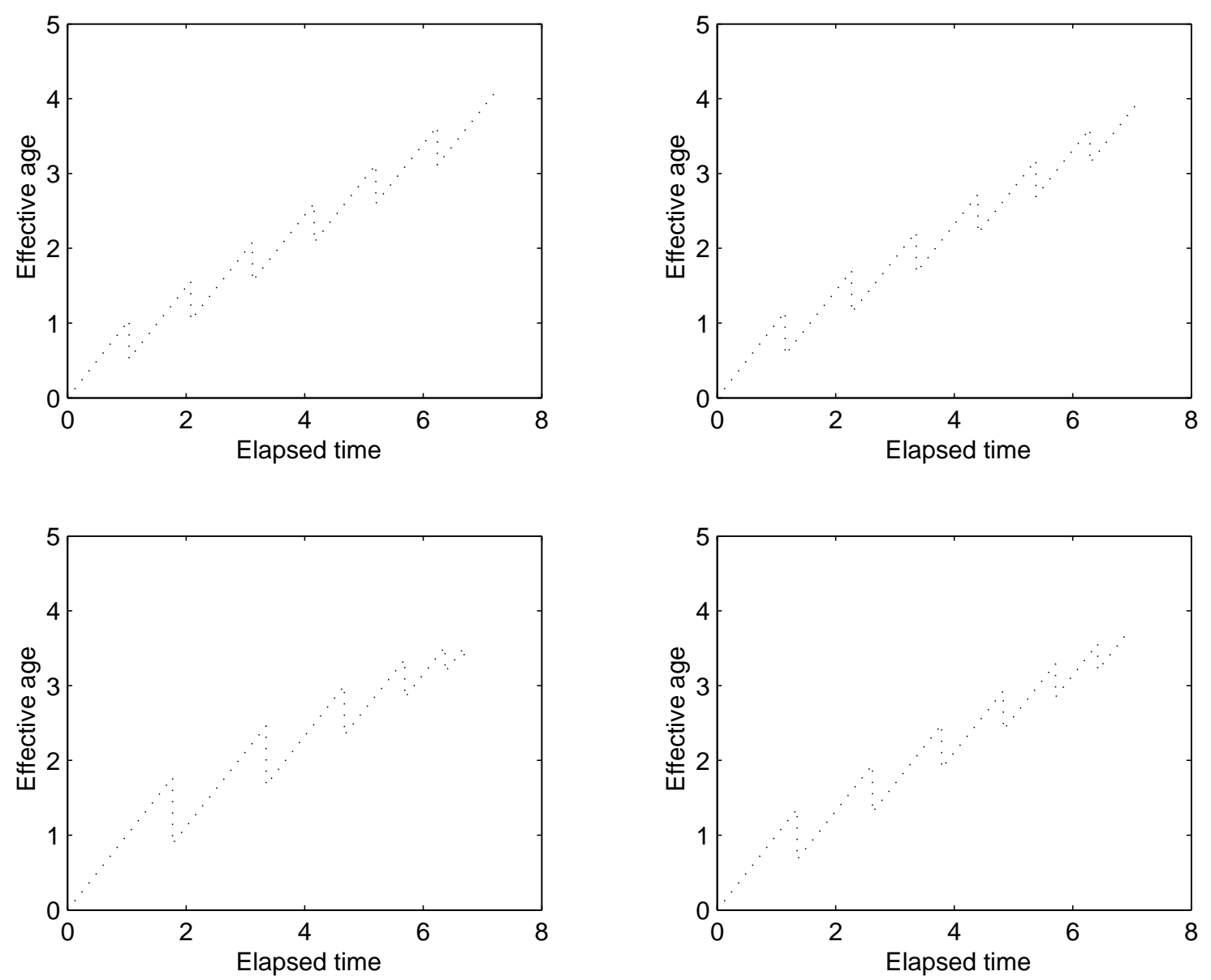

Figure 3: Effective age in type 1 schedules for $\hat{a}=1,1.01,1.025,1.05$ (clockwise from top left)

The effective age plots for the four solutions in Figure 3 all have similar form, but a closer inspection shows that each increase in $\hat{a}$ leads to the first PM being performed 
later. However, the subsequent inter-PM times, $x_{2}, x_{3} \ldots$, become progressively shorter when $\hat{a}>1$ and so $t_{N}^{*}$ decreases as $\hat{a}$ increases. We note, in particular, that when $\hat{a}=1.05$ (bottom left graph) there are effectively only five PMs (rather than six as in the other three cases) because the optimization has forced the last inter-PM interval to zero.

Table 3 and Figure 4 give similar information about schedules obtained with the type 2 aging model for varying $\hat{a}$ values. They show that the first type 2 PM typically occurs much later than in any of the type 1 schedules and that this difference becomes more marked as $\hat{a}$ increases. Moreover, the type 2 inter-PM times, $x_{2}, x_{3}, \ldots$, decrease more rapidly those in the type 1 solutions. This is shown in the sub-plots in Figure 4: the type 2 schedule with $\hat{a}=1.75$ effectively only uses 4 PMs rather than 6 because the optimum values of $x_{5}, x_{6}$ and $x_{7}$ are all zero. Table 3 also shows how $t_{N}^{*}$ decreases and $C^{*}$ increases for type 2 schedules as $\hat{a}$ gets larger. If we recall that the optimum mean cost without $P M$ is 190.1 then we can see from the second column of Table 3 that, if $\hat{a}$ is large, there may be little to be gained by using a type 2 optimal PM schedule.

\begin{tabular}{c|c|rrrrrrr|c}
$\hat{a}$ & $C^{*}$ & \multicolumn{7}{|c|}{$x_{k}^{*}$} & $t_{N}^{*}$ \\
\hline 1.1 & 132.8 & 2.6 & 1.05 & 0.95 & 0.86 & 0.77 & 0.7 & 1.24 & 8.18 \\
1.25 & 156.8 & 2.29 & 0.96 & 0.76 & 0.60 & 0.46 & 0.36 & 0.52 & 6.95 \\
1.4 & 173.8 & 3.89 & 0.81 & 0.56 & 0.39 & 0.26 & 0.17 & 0.16 & 6.24 \\
1.5 & 181.3 & 4.24 & 0.68 & 0.44 & 0.28 & 0.17 & 0.10 & 0.02 & 5.93 \\
1.6 & 186.2 & 4.56 & 0.54 & 0.33 & 0.19 & 0.11 & 0.0 & 0.0 & 5.73 \\
1.75 & 190.1 & 4.99 & 0.34 & 0.18 & 0.05 & 0.0 & 0.0 & 0.0 & 5.56
\end{tabular}

Table 3: Optimal costs for type 2 schedules and varying $\hat{a}$

\subsection{Effect of the parameter $\hat{b}$}

A decrease in the parameter $\hat{b}$ appearing in (12) represents an increase in the efficiency of each PM. Hence we expect it to produce a decrease in $C^{*}$ and an increase in $t_{N}^{*}$. To see how optimum PM schedules change to achieve this, we suppose $\gamma_{r}$ and $\gamma_{m}$ are as given in (13) and we fix the values $\hat{a}=1.025$ for the type 1 model and $\hat{a}=1.25$ for the type 2 model. We use the quadratic model (10) for $H(t)$ and consider how the optimum PM schedules vary with $\hat{b}$ in the case when $N=7$. We illustrate schedules by showing the inter-PM times, $x_{1}, x_{2}, \ldots, x_{7}$. Figures 5 and 6 indicate the occurrence of each PM by a symbol along a horizontal time line; the height of each of these time lines indicates the 

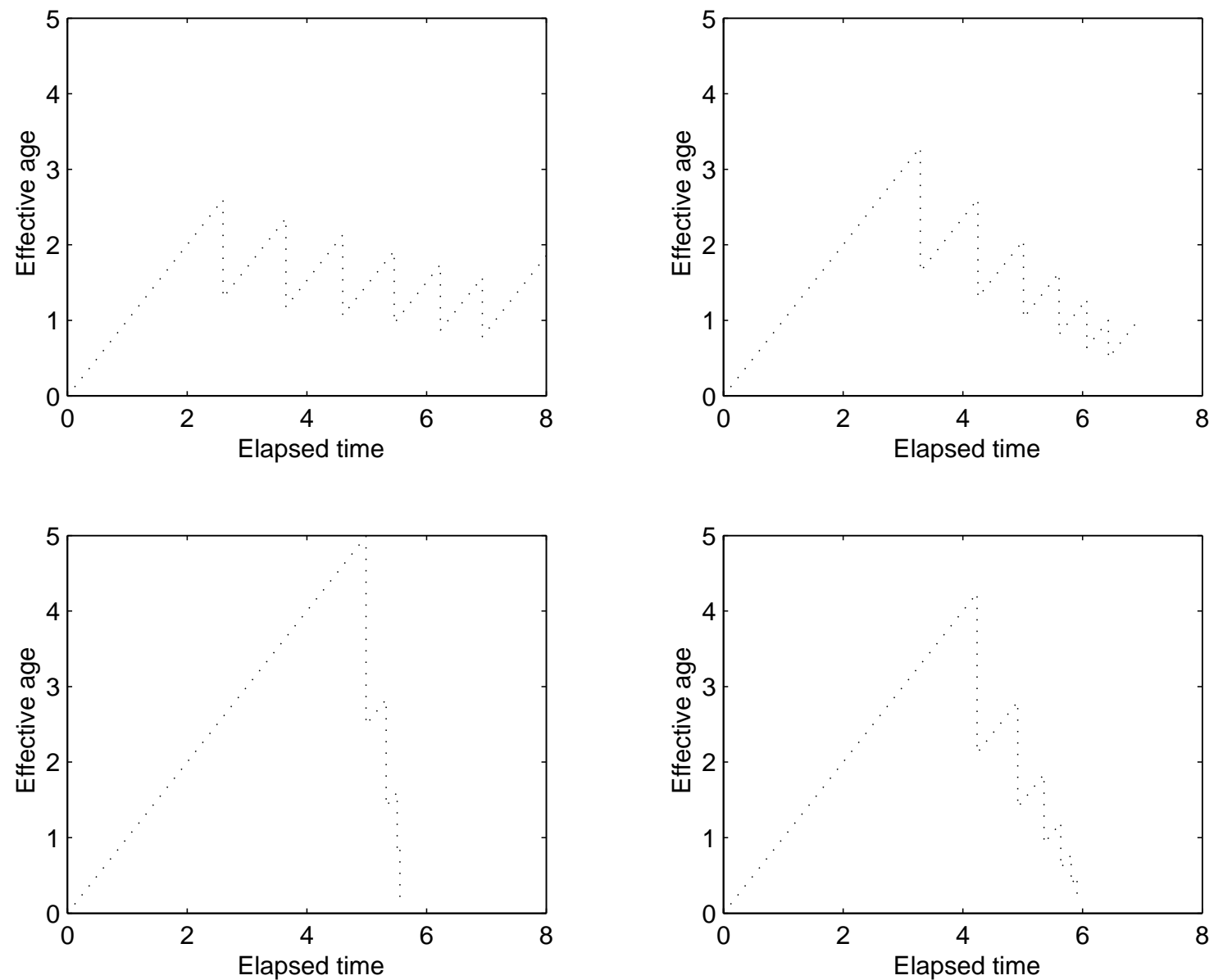

Figure 4: Effective age in type 2 schedules for $\hat{a}=1.1,1.25,1.5,1.75$ (clockwise from top left)

corresponding mean cost, $C^{*}$. The inter-PM intervals tend to decrease more rapidly as $\hat{b}$ increases. This is true for both effective age models: but the type 1 schedules apply PM rather more uniformly than the type 2 schedules.

We now consider the case when the $b_{k}$ are not constant but increase towards 1 as $k$ increases. This represents a law of diminishing returns in the effectiveness of PM over equipment operating life that could be an alternative to the use of the parameters $A_{j}$ in (5). We consider a model where

$$
b_{1}=0.5 \text { and } b_{k}=b_{k-1}^{\alpha} \quad \text { where } \quad \alpha<1 .
$$

Figures 7 and 8 show results for varying $\alpha$ with $\gamma_{r}$ and $\gamma_{m}$ from (13) and using $\hat{a}=1$ for both type 1 and type 2 schedules. We see that a more rapid decline in the efficacy of PM 


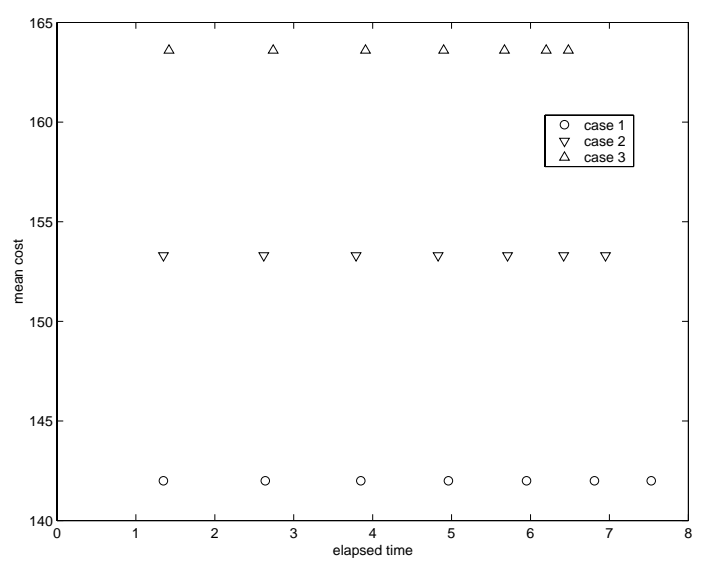

Figure 5: Type 1 schedules for varying $\hat{b}(1: \hat{b}=0.4,2: \hat{b}=0.5,3: \hat{b}=0.6)$

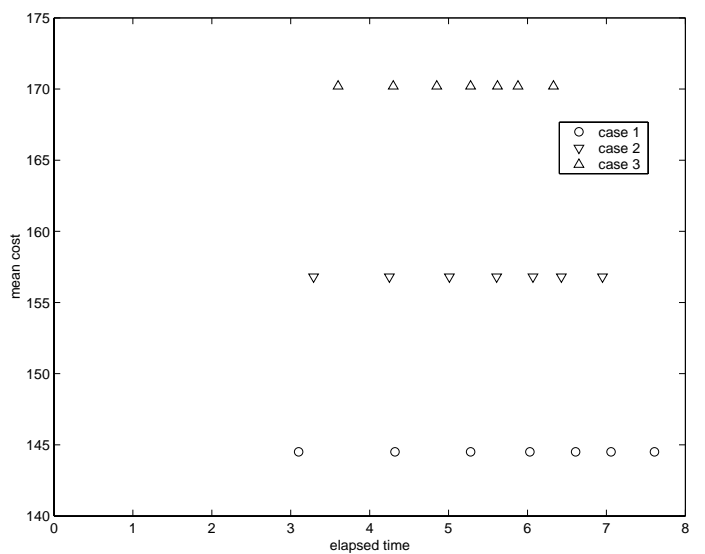

Figure 6: Type 2 schedules for varying $\hat{b}(1: \hat{b}=0.4,2: \hat{b}=0.5,3: \hat{b}=0.6)$

- i.e., a decrease in $\alpha$ in (14) - causes the first one or two inter-PM times to increase; but subsequent $x_{k}$ then typically decrease so there is an overall reduction in time to replacement and mean cost. This remark applies to both type 1 and type 2 solutions (although Figure 8 shows that type 2 schedules differ from type 1 in having $x_{N}>x_{N-1}$ ).

Comparing the middle row of Figure 5 with all three rows in Figure 8 suggests that type 2 schedules in which $b_{k}$ increases from one PM to the next bear some resemblance to type 1 schedules obtained when $b_{k}$ is constant. 


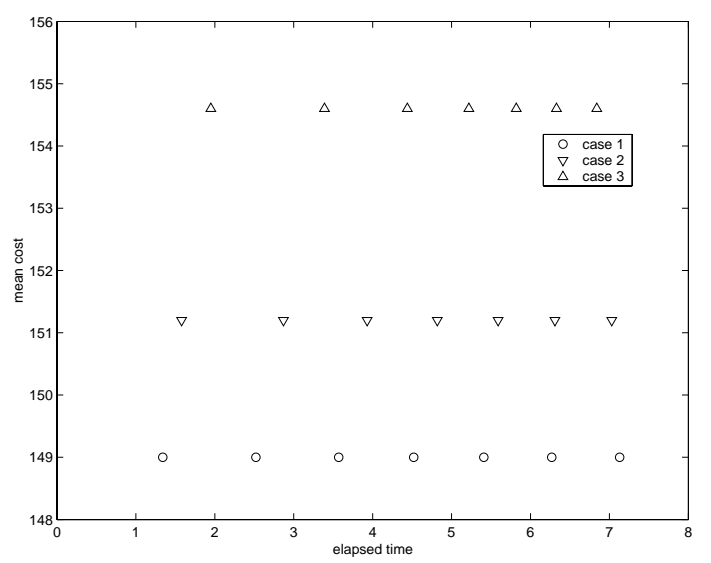

Figure 7: Type 1 schedules for $b_{k}=b_{k-1}^{\alpha}(1: \alpha=0.95,2: \alpha=0.9,3: \alpha=0.8)$

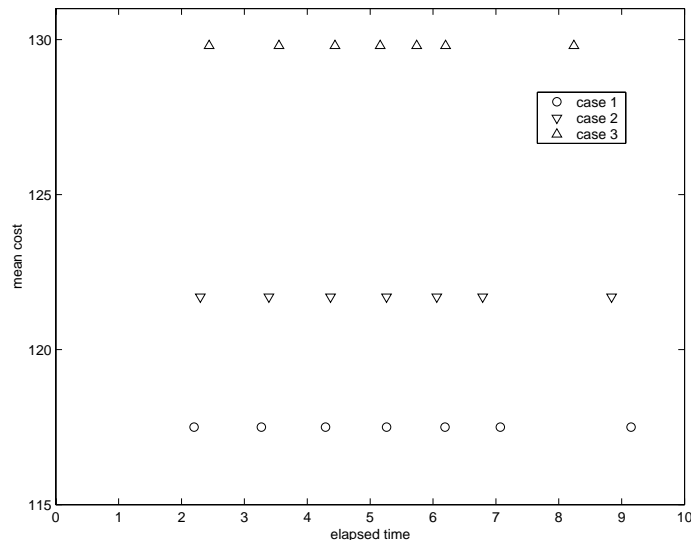

Figure 8: Type 2 schedules for $b_{k}=b_{k-1}^{\alpha}(1: \alpha=0.95,2: \alpha=0.9,3: \alpha=0.8)$

\subsection{Effect of changes in repair and replacement cost}

We now consider how optimal PM schedules are affected by the relative costs of replacement and minimal repair, $\gamma_{r}$ and $\gamma_{m}$. Results shown in Figure 9 are for type 1 schedules using $\hat{b}=0.5, \hat{a}=1.025, \gamma_{r}=500, N=7$ with varying $\gamma_{m}$. Not unexpectedly, $C^{*}$ decreases and $t_{N}^{*}$ increases as $\gamma_{m}$ gets smaller. Specifically, as $\gamma_{m}$ is halved, the inter-PM times all increase by a factor of about $\sqrt{2}$.

The type 2 schedules in Figure 10 also use the values $\hat{b}=0.5, \gamma_{r}=500, N=7$ with $\hat{a}=1.25$. These schedules are quite different from those in Figure 9. However, the effect of decreasing minimal repair costs is qualitatively the same as for the type 1 model because the inter-PM times (and hence $t_{N}^{*}$ ) increase by about $\sqrt{2}$ when $\gamma_{m}$ is halved. 


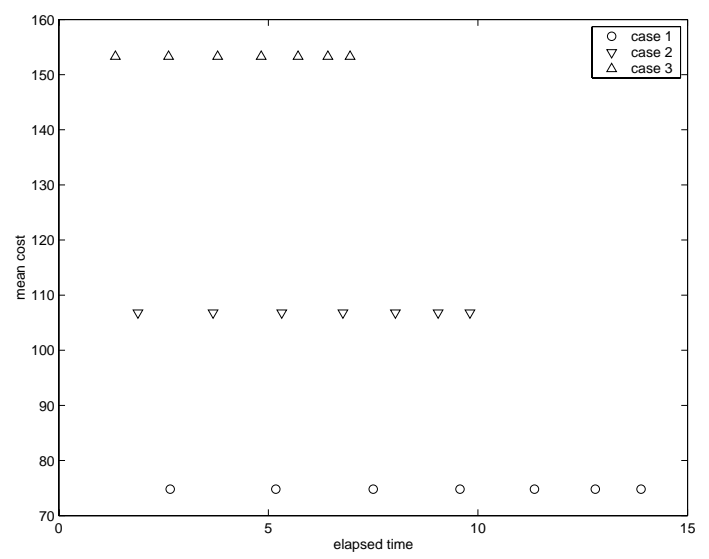

Figure 9: Type 1 schedules for varying $\gamma_{m}\left(1: \gamma_{m}=25,2: \gamma_{m}=50,3: \gamma_{m}=100\right)$

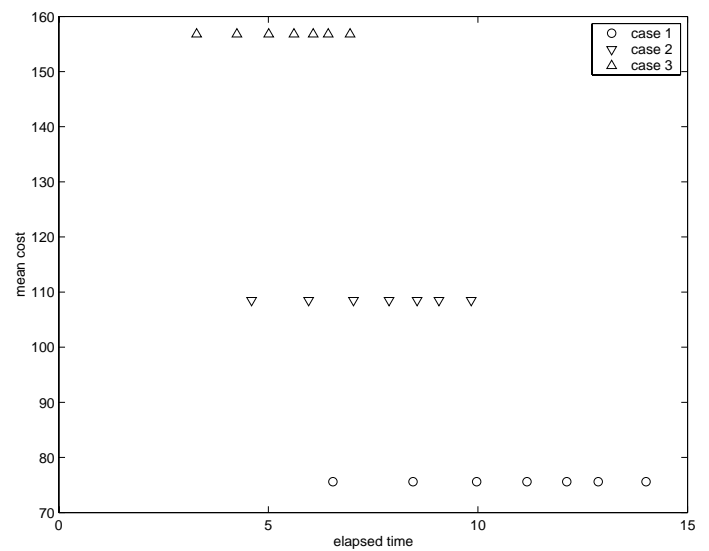

Figure 10: Type 2 schedules for varying $\gamma_{m}\left(1: \gamma_{m}=25,2: \gamma_{m}=50,3: \gamma_{m}=100\right)$

We now consider the effect of changes in replacement cost $\gamma_{r}$. Using the type 1 age model with parameters $\hat{b}=0.5, \hat{a}=1.025, \gamma_{m}=100$ and $N=7$, the optimum mean cost varies with $\gamma_{r}$ as follows:

When $\gamma_{r}=500, C^{*}=153.3$;

When $\gamma_{r}=1000, \quad C^{*}=213.1$;

When $\gamma_{r}=2000, \quad C^{*}=297.8$.

The PM schedules which produce these costs are essentially the same as those in Figure 9. In other words, doubling replacement cost has virtually the same effect on the optimal PM strategy as halving minimal repair cost. This observation follows from the roles played by $\gamma_{r}$ and $\gamma_{m}$ in the cost function (7) and so it is also true for type 2 schedules 


\subsection{Effect of number of PMs}

We have already noted (see Table 1) that the optimum mean cost depends on the number of PMs that are performed. Figure 11 illustrates this for the type 1 age model with $\hat{b}=0.5, \hat{a}=1.025, \gamma_{r}=500, \gamma_{m}=100$. Recalling that the minimum mean cost without $\mathrm{PM}$ is about 190.1, we see that the biggest improvement comes in the $N=2$ case where a single, well-placed, PM reduces the mean cost by about $12 \%$. Further PMs also produce improvements, but the benefits of each extra PM becomes smaller.

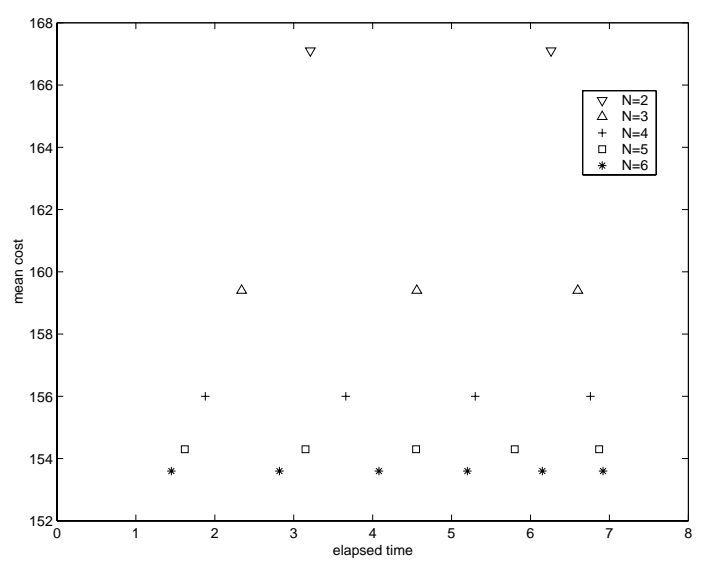

Figure 11: Type 1 schedules for varying $N$

Similar behaviour is observed for type 2 schedules. Figure 12 was obtained using values $\hat{b}=0.5, \hat{a}=1.25, \gamma_{r}=500, \gamma_{m}=100$ and it shows that reductions in mean cost (and the change in distribution of PMs) become relatively smaller as $N$ gets larger.

If $N$ is increased beyond the range illustrated in Figures 11 and 12 then type 1 and type 2 solutions behave in different ways. The optimal type 1 schedule with $N=8$ adds one further short interval, $x_{8} \approx 0.32$, but this does not produce any reduction in mean cost compared with the $N=7$ solution. The type 1 schedule with $N=9$ adds a further interval $x_{9} \approx 0.13$ which produces a small increase in mean cost compared with the $N=7$ case. Type 1 solutions with $N \geq 10$ then remain effectively the same as for $N=9$ with $x_{10}, x_{11}, .$. all being zero. In other words, the type 1 model identifies six PMs as being the optimal choice.

The type 2 model, on the other hand, suggests it is beneficial to perform more than six PMs. Type 2 solutions show continuing (but small) improvements in $C^{*}$ as $N$ increases from 7 to 11 . The schedules for $N=12,13$ give higher mean cost than when $N=11$ and 


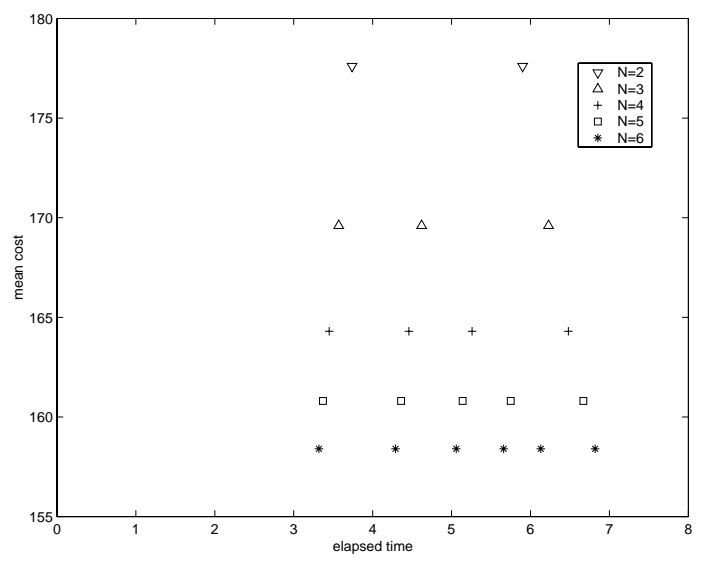

Figure 12: Type 2 schedules for varying $N$

feature some very short inter-PM intervals (e.g. $x_{12} \approx 0.05, x_{13} \approx 0.02$ ). For $N \geq 14$ the solutions stay effectively the same as for $N=13$ with all subsequent $x_{k}$ being set to zero.

\subsection{Effect of changing failure rate model}

The results in preceding sections were obtained using the quadratic model (10) for $H(t)$. Repeating the calculations using the cubic model (11), made almost no significant difference to the results: the optimal inter-PM times sometimes changed only in the second or third digit. Use of the cubic model does remove the small curiosity, noted in section 5.2 , that all the $x_{k}$ are equal in type 1 sschedules with $\hat{a}=1$; but the inter-PM times are still effectively equal for practical purposes. In the examples discussed in the preceding subsection, the cubic model of $H(t)$ indicates that the optimal number of PMs with type 2 aging is twelve as opposed to ten for the quadratic model. However the variations in mean cost for $10 \leq N \leq 13$ are so small that this change does not seem to be of much significance.

\subsection{Effect of non-maintainable failure modes}

In practice, $\mathrm{PM}$ operations (like cleaning, oiling and adjustment) can reduce the frequency of mechanical failures. However there may be no corresponding PM interventions that will extend the life of sealed electronic units - that is, some parts of a system may be nonmaintanable. If $h_{a}(t)$ denotes the hazard rate of maintainable failure modes and $h_{b}(t)$ is the hazard rate for non-maintainable ones then the number of failures occurring between 
times $t_{k-1}$ and $t_{k}$ is

$$
H_{k-1}\left(t_{k}\right)=\int_{y_{k-1}^{+}}^{y_{k}} A_{k-1} h_{a}(y) d y+\int_{t_{k-1}}^{t_{k}} h_{b}(t) d t .
$$

That is, some failures depend on effective age and some depend on calendar age. (In practice, it may not be easy to make clear distinctions between failure modes.) Thus the total number of failures occurring between $t=0$ and $t=t_{k}$ would be

$$
H\left(t_{k}\right)=\sum_{j=1}^{k-1} H_{j-1}\left(t_{j}\right)
$$

which gives a similar expression to (5), namely

$$
H\left(t_{k}\right)=\int_{0}^{y_{1}} h_{a}(y) d y+\sum_{j=1}^{k-1}\left\{\int_{y_{j}^{+}}^{y_{j+1}} A_{j} h_{a}(y) d y\right\}+\int_{0}^{t_{k}} h_{b}(t) d t .
$$

If no PM were performed the number of failures between $t=0$ and $t=t_{k}$ would be

$$
H\left(t_{k}\right)=\int_{0}^{t_{k}} h_{a}(t) d t+\int_{0}^{t_{k}} h_{b}(t) d t
$$

We now consider PM schedules in the presence of non-maintainable failure modes. We shall assume that the cumulative failure functions $H(t)$ are given by (10) and (11) but that a fraction $\theta$ of the failures are due to non-maintainable elements. For case A, the cumulative failure rate is

$$
H(t)=0.1676 t^{2}+0.0704 t
$$

and so the failure rate $h(t)$ in (5) is obtained by differentiation as

$$
h(t)=0.3352 t+0.0704 .
$$

We now suppose that this splits into maintainable and non-maintainable failure rates

$$
h_{a}(t)=(1-\theta)(0.3352 t+0.07), \quad h_{b}(t)=\theta(0.3352 t+0.07) .
$$

Obviously there would be similar definitions of $h_{a}$ and $h_{b}$ for Case B. If we substitute such expressions for $h_{a}$ and $h_{b}$ in (15) we obtain the number of failures occurring between the $(k-1)$-th and $k$-th PMs; and this, in turn, feeds into the cost function (7).

Tables 4 and 5 are obtained by introducing non-maintainable failures into the system used for previous numerical examples. These tables show that - as might be expected - 


$$
\begin{array}{cc|cc|cc}
\theta=0 & \multicolumn{2}{|c|}{\theta=0.2} & \multicolumn{2}{c}{\theta=0.4} \\
C^{*}, \quad t_{N}^{*} & C^{*}, & t_{N}^{*} & C^{*}, & t_{N}^{*} \\
\hline 153.3, & 6.94 & 161.6, & 6.56 & 169.6, & 6.24
\end{array}
$$

Table 4: Effects of $\theta$ on type 1 schedules $\left(\hat{b}=0.5, \hat{a}=1.025, \gamma_{r}=500, \gamma_{m}=100 \& N=7\right)$

\begin{tabular}{|c|c|c|}
\hline$\theta=0$ & $\theta=0.2$ & $\theta=0.4$ \\
\hline$C^{*}, \quad t_{N}^{*}$ & $C^{*}, \quad t_{N}^{*}$ & $C^{*}$ \\
\hline $156.8,6.95$ & $164.5,6.57$ & $171.7,6.24$ \\
\hline
\end{tabular}

Table 5: Effects of $\theta$ on type 2 schedules $\left(\hat{b}=0.5, \hat{a}=1.25, \gamma_{r}=500, \gamma_{m}=100 \& N=7\right)$

the beneficial effect of PM is diminished as $\theta$ gets larger. Hence the optimal mean cost rises (because more failures will occur) and the optimal lifetime decreases.

This way of modelling non-maintainable failure modes affects both type 1 and type 2 schedules in a similar way. As $\theta$ increases then all the inter-PM times decrease in quite a uniform manner: for the particular system in these examples, each increase of 0.2 in $\theta$ causes all the optimal $x_{k}$ to decrease by about $5 \%$.

\section{Conclusions \& discussion}

In this paper we have outlined two forms of the effective-age approach to modelling preventive maintenance. In the type 1 model, the $k$-th PM only reduces the aging that has occurred since the $(k-1)$-th PM; but in the type 2 model the $k$-th PM makes a cumulative reducation on all aging since the equipment entered service. Both types of age model can be used to compute a mean cost function; and optimal PM schedules are then obtained by adjusting inter-PM intervals to minimize this function.

We have computed optimal PM schedules based on both type 1 and type 2 aging. Our results show that type 1 and type 2 schedules can be quite different from each other. Type 1 schedules require PMs to be fairly uniformly distributed (see Figure 3). In type 2 schedules, the spread of inter-PM times is greater, with a relatively long delay before the first PM while subsequent PMs quickly become relatively closer together (see Figure 4). Furthermore, the type 2 aging model admits optimum schedules which cause effective age to decrease steadily over the operating life. This does not seem to be the case with 
type 1 solutions. The fact that the type 2 solutions allow equipment to become effectively younger and younger in spite of increasing calendar age means that the $A_{j}$ parameters in (5) are important in ensuring that type 2 solutions do not become unrealistic. The type 1 model is intuitively more convincing in the way that each PM is assumed only to counteract the most recent deterioration in the state of the system.

Our numerical tests have also shown how type 1 and type 2 optimal schedules respond to changes in the effectiveness of PM (as given by $b_{k}$ and $A_{j}$ in (2), (4) and (5)) and to changes in the relative costs of repair and system replacement.

For any particular case there will be an optimum number of PMs - i.e., one which yields the least value of the cost function (7). Our tests indicate that this optimum number is likely to be higher for type 2 schedules than for type 1 . However, it also appears that the optimum mean cost, $C^{*}$, becomes less sensitive to the number of PMs as $N$ gets larger.

Finally we have shown how both type 1 and type 2 optimum PM schedules can change if the system is regarded as having both maintainable and non-maintainable components.

Two modifications to the PM scheduling problem may be worth attention in future. Just as we may distinguish between maintainable and non-maintainable failure modes so we might subdivide the maintainable modes into type 1 and type 2 classes. PM functions such as lubrication and adjustment can reasonably be expected to reduce effective age in a type 1 manner; but where PM actually involves some degree of replacement then the type 2 model could be appropriate.

A second observation is that maintenance does not just reduce the occurrence of failures but also makes equipment operate more efficiently. Hence we could extend the mean cost function to reflect operating costs as well as repair costs. We would expect, for instance, a newer system to be more fuel efficient than an older one; and so effective age could appear in an expression for running costs just as it does in the expression for failure rate. Gathering data for formulating running cost as a function of age could, in practice, be as challenging as the task of modelling the cumulative failure rate function $H(t)$. Nonetheless, it would be worth the attempt, so that PM scheduling could be based on a more complete representation of lifetime costs.

\section{References}

[1] M Bartholomew-Biggs, B Christianson and M Zuo (2006). Optimizing preventive maintenance models, Computational Optimization and Applications, 35(2), 261-279. 
[2] J S Dagpunar (1998). Some properties and computational results for a general repair process. Naval Research Logistics Quarterly, 45(4), 391-405.

[3] R Dekker (1996). Applications of Maintenance Optimization Models: A Review and Analysis. Reliability Engineering and System Safety, 51, 229-240.

[4] L. Doyen \& O. Gaudoin (2004), Classes of imperfect repair models based on reduction of failure intensity on virtual age, Reliability Engineering and System Safety, 84, 45-56.

[5] W. Kahle (2006), Optimal maintenance policies on incomplete repair models, Reliability Engineering and System Safety, 92, 563-565.

[6] M Kijima, H Marimura and Y Suzuki (1988). Periodical Replacement Problem Without assuming Minimal Repair. European Journal of Operational Research, 37(2), 194203.

[7] M Kijima (1989). Some results for repairable systems with general repair. Journal of Applied Probability 26, 89-102.

[8] D Lin, M J Zuo and R C M Yam (2001). Sequential imperfect preventive maintenance models with two categories of failure modes. Naval Research Logistics 48, 172-183.

[9] J McCall (1965). Maintenance Policies for Stochastically Failing Equipment: A Survey. Management Science, 11, 493-524.

[10] R.Pascual and J.H.Ortega (2006), Optimal replacement and overhaul decisions with imperfect maintenance and warranty contracts, Reliability Engineering and System Safety, 91, 241-248.

[11] H Pham and H Wang (1996) Imperfect Maintenance. European Journal of Operational Research, 94, 425-438

[12] W Pierskalla and J Voelker (1976). A Survey of Maintenance Models: The Control and Surveillance of Deteriorating Systems. Naval Research Logistics Quarterly, 23, 353388.

[13] H Wang (2002). A survey of maintenance policies of deteriorating systems. European Journal of Operational Research 139, 469-489.

[14] C Valdez-Flores (1989). Survey of Preventive Maintenance Models for Stochastically Deteriorating Single-Unit Systems. Naval Research Logistics, 36, 419-446. 